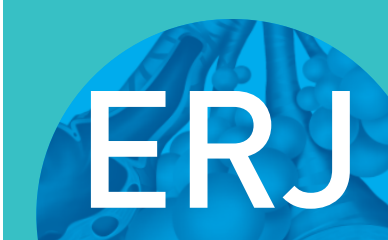

open research

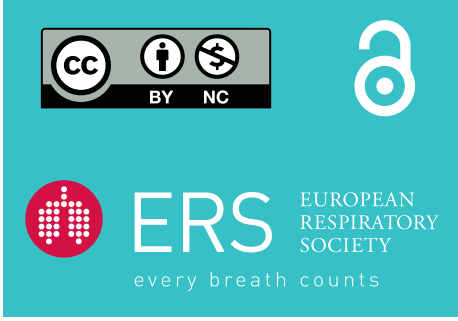

\section{Novel mouse model of indwelling pleural catheter in mice with malignant pleural effusion}

\author{
To the Editor:
}

Malignant pleural effusion (MPE) is an indicator of advanced stage malignancy. There are an estimated 150000 to 200000 cases of MPE diagnosed annually in the USA [1]. During the past 10 years, the types of cancer treatment and pleural procedures available for MPE have expanded leading to improved stratification and better individualisation of treatment [2]. A recently published prognostic score (PROMISE score) improved prediction of the 3 month risk of death in patients with MPE, thereby improving the selection of appropriate management strategies [3].

Indwelling pleural catheters (IPC) have become a mainstay of care and palliation in individuals with MPE [4]. Intrapleural therapy for malignancy is a burgeoning area of research and IPCs provide direct access to the pleural space with the potential of testing novel treatment agents $[5,6]$. To date, there has not yet been a reliable mouse model of MPE utilising IPC. Such a model could be used to translate in vivo experiment results to future clinical trials testing new agents in MPE. The method highlighted below is the first experimental IPC model in MPE.

For the IPC mouse model, adult wild type C57BL/6 mice (in-house breeding) were used. Experimental mice were matched for sex, weight $(20-25 \mathrm{~g})$ and age (6-12 weeks). Mice were sacrificed 14 days post intrapleural delivery of cancer cells.

Lewis lung carcinoma (LLC) cells were cultured in Gibco Dulbecco's modified eagle medium (Thermofisher Scientific, Waltham, MA, USA) supplemented with $10 \%$ fetal bovine serum and $100 \mathrm{mg} \cdot \mathrm{L}^{-1}$ penicillin/ streptomycin at $37^{\circ} \mathrm{C}$ in $5 \% \mathrm{CO}_{2}-95 \%$ air. Cells were resuspended by trypsinisation prior to counting. Two different and independent researchers counted the cells using a haemocytometer.

MPE was formulated by intrapleural injection of LLC cells into the pleural space. Mice were first anaesthetised with isoflurane at 3-L per minute flow of oxygen by calibrated vaporiser. Once anaesthetised, the animal fur on the right thoracic flank posterior to the forelimb was shaved using electric clippers. The shaved mouse was then placed into a nose cone for ongoing anaesthesia, and placed on top of a warm circulating water blanket throughout the procedure to prevent hypothermia. With the mouse in the left lateral decubitus position, the right thorax was coated in iodine, and the subcutaneous skin was incised with surgical scissors and retracted. Further dissection was performed to reveal the bony thorax and parietal pleura, though the pleura remained intact. Under direct visualisation, and using a 1cc syringe with $21 \mathrm{G}$ needle, a suspension of 250000 LLC cells in $100 \mu \mathrm{L}$ PBS was injected into the thoracic space. Following injection, the subcutaneous tissues were secured into anatomic position with topical tissue adhesive. Mice were removed from sedation and monitored until fully recovered.

From previous published data, we determined that approximately 7-9 days are required for development of MPE from time of LLC intrapleural injection [7]. Therefore, on day 7, intrapleural catheters (Instech Laboratories, Plymouth Meeting, PA, USA) (figure 1a) were positioned to drain pleural effusions. The mice were anaesthetised with isoflurane and again shaved in the same manner as required for the initial

$@$ ERSpublications

This novel mouse model mimics malignant pleural effusion drainage using an indwelling pleural catheter in humans, and provides direct access to the pleural space potentially enabling the testing of intrapleural therapies in the treatment of MPE. bit.ly/2W2kzO0

Cite this article as: Merrick C, Sherrill T, Kanellakis NI, et al. Novel mouse model of indwelling pleural catheter in mice with malignant pleural effusion. ERJ Open Res 2019; 5: 00226-2018 [https:// doi.org/10.1183/23120541.00226-2018].

Copyright $\odot$ ERS 2019. This article is open access and distributed under the terms of the Creative Commons Attribution Non-Commercial Licence 4.0. 
injection. Following shaving, mice were placed under a nose cone in the left lateral decubitus position on top of a warm circulating water blanket. Similarly to initial injection, the subcutaneous tissue was dissected after the skin was sterilised with iodine. Once the thorax was exposed, a small puncture was made through the seventh rib space in the mid axillary line using a $16 \mathrm{G}$ blunt tipped needle. Immediately following puncture, the needle was removed and polyurethane tubing (PU-40) was advanced approximately $1.5 \mathrm{~cm}$ into the pleural space. Simultaneously, a sharp tipped $16 \mathrm{G}$ needle was punctured through the fur on the animals dorsum, midway between scapulae, and advanced from point of insertion, through subcutaneous tissue toward the catheter insertion site in the right flank. The proximal end of the PU-40 tubing was then passed over the needle and out between the scapulae. The needle was then removed, leaving the pleural catheter tunnelled through the subcutaneous space (figure 1b). The proximal end of the catheter was anchored at the insertion site with application of GLUture (World Precision Instruments, Hitchin, UK) and the distal end was sutured into place between the scapulae with ethilon 5-0 monofilament suture. Finally, the area of dissection was closed with approximation of tissue and application of topical tissue adhesive. Of note, prior to catheter insertion, three full thickness punctures were made at the distal end of the catheter using a $22 \mathrm{G}$ needle to allow enhanced catheter flow. Catheters were capped with a $22 \mathrm{G}$ syringe accessible port, which was placed on the proximal end of the catheter prior to pleural insertion in order to prevent tension pneumothorax.

GraphPad PRISM 7 (GraphPad Software, San Diego, CA, USA) was used for statistics and to generate graphs.

This study (protocol M1700024-00-AN1) was approved by the Institutional Animal Care and Use Committee in May 2017.

Following catheter placement, fluid aspiration was performed while mice were still under general anaesthesia with isoflurane. This was achieved with a $22 \mathrm{G}$ syringe connected to an adapter which was compatible with the port on the proximal end of the catheter. Aspirate volume was variable based on the degree of MPE and degree of bulky tumour at the distal end of the catheter. The puncture sites at the distal end of the catheter did improve the ability to aspirate fluid, even when the catheter was surrounded
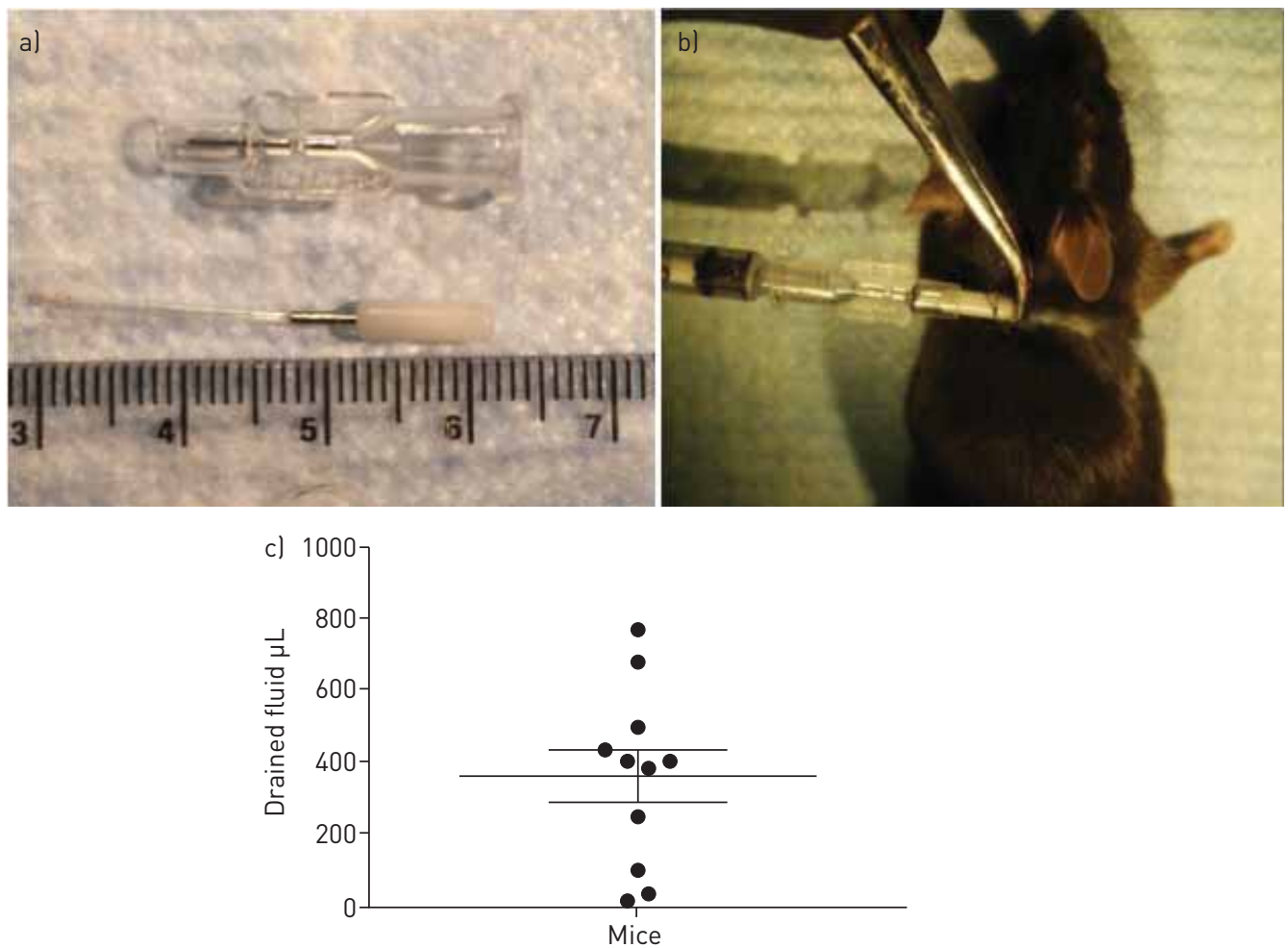

FIGURE 1 a) The mouse indwelling pleural catheter. The catheter is composed of polyurethane tubing and a syringe accessible port, with syringe adapter. b) Mouse with the intrapleural catheter tunnelled through subcutaneous tissue, with a $22 \mathrm{G}$ syringe-accessible port on the distal end of the catheter. The port is sutured to skin. c) Pleural fluid drained from mice. The scatterplot shows the mean land standard error of mean) volume of pleural fluid drained from 11 mice. Eight mice were excluded from the analysis because of technical issues. 
by bulky tumour but did not, however, fully obviate the problem. Following fluid aspiration, anaesthesia was reversed and animals were observed until full recovery.

The pleural space was again aspirated on day 14 or earlier if mice became moribund. The mean overall volume of aspirate was $188 \mu \mathrm{L}$ and ranged from 0 to $770 \mu \mathrm{L}(\mathrm{n}=19)$. Eight mice were excluded from further analysis because of technical issues (peritoneal tumour indicating faulty injection $(\mathrm{n}=3)$, catheter was pulled out $(n=1)$, tumour encasing (and blocking) catheter $(n=2)$, and minimal effusion $(n=2)$ ). Figure 1c shows the overall fluid drained from the other 11 mice: mean 359.1 (SD 246.9, SEM 74.5, 95\% CI 193.2-525) $\mu \mathrm{L}$. On day 14, mice were sacrificed in a $\mathrm{CO}_{2}$ inhalational chamber and final aspiration was performed. After final aspiration, mice were dissected and the pleural space was exposed. This typically demonstrated bulky pleural tumour with minimal residual effusion. Furthermore, residual effusion with catheter envelopment by tumour was found in two mice, leading to inability to aspirate the catheter.

Following fluid aspiration, any potential agent could be injected through the pleural catheter by means of the syringe accessible port (PinPort; Instech Laboratories); therefore, this model allows both effusion aspiration and potentially intrapleural drug delivery. A typical volume of $100 \mu \mathrm{L}$ PBS was well tolerated by the animals.

Most of the mice were sacrificed because they became moribund by days 13-16 $(n=14)$. In the other mice, some were well at day 14 therefore were sacrificed at a later stage on day $18(n=2)$ and day $22(n=1)$, and others died due to unforeseen reasons on day $10(n=1)$ and day $12(n=1)$.

This is a novel mouse model of MPE, which seeks to recapitulate the human experience by using tunnelled IPCs. We demonstrate that the establishment of IPC is possible in a mouse model of MPE. This model allows both effusion aspiration as well as delivery of intrapleural agents, as demonstrated with intrapleural PBS.

Limitations of this model include the occasional blockage (in two (10.5\%) out of 19 mice) of the IPC by tumour bulk leading to limited MPE aspiration, and the limited development of MPE in a further two (10.5\%) mice. The first limitation can be overcome by generating additional holes in the intrapleural part of the catheter. The second limitation is a phenomenon also seen in humans where some patients develop MPE and others with identical tumour subtype and staging do not. Further studies using this model should take into account the potential small number of mice that may not develop large volumes of MPE. It is expected that with further experience and standardisation of the protocol, the rates of development of peritoneal tumour (indicating faulty injection) and catheter fall out will be significantly reduced.

This model can be used as a platform for a broad array of therapeutic pleural experiments for both translational research and pre-clinical studies aiming to assess the safety of new agents.

Christopher Merrick ${ }^{1}$, Taylor Sherrill ${ }^{1}$, Nikolaos I. Kanellakis ${ }^{2,3}$, Rachelle Asciak ${ }^{2,3}$, Georgios T. Stathopoulos ${ }^{4,5}$, Fabien Maldonado $^{1}$, Najib M. Rahman ${ }^{2,3}$, Timothy Blackwell ${ }^{1}$ and Ioannis Psallidas $2,3,6$

${ }^{1}$ Vanderbilt University Medical Center, Nashville, TN, USA. ${ }^{2}$ Oxford Centre for Respiratory Medicine, Oxford, UK. ${ }^{3}$ Laboratory of Pleural and Lung Cancer Translational Research, Nuffield Dept of Medicine, University of Oxford, Oxford, UK. ${ }^{4}$ Comprehensive Pneumology Center and Institute for Lung Biology and Disease, University Hospital, Ludwig-Maximilian University, Helmholtz Center Munich, Member of the German Center for Lung Research, Munich, Germany. ${ }^{5}$ Laboratory for Molecular Respiratory Carcinogenesis, Dept of Physiology, Faculty of Medicine, University of Patras, Patras, Greece. ${ }^{6}$ Lungs for Living Research Centre, UCL Respiratory, University College London, London, UK.

Correspondence: Ioannis Psallidas, Centre for Respiratory Medicine, 5 Rayne Building, University Street, London, WC1E 6JF, UK. E-mail: ipsallidas@ucl.ac.uk

Received: Nov 292018 | Accepted after revision: March 312019

Conflict of interest: C. Merrick has nothing to disclose. T. Sherrill has nothing to disclose. N.I. Kanellakis has nothing to disclose. R. Asciak has nothing to disclose. G.T. Stathopoulos has nothing to disclose. F. Maldonado has nothing to disclose. N.M. Rahman has received consultancy fees from Rocket Medical UK, outside the submitted work. T. Blackwell has nothing to disclose. I. Psallidas works as a Medical Science Director in AstraZeneca in a non-related field.

Support statement: The authors acknowledge support from the US Department of Veterans Affairs. Funding information for this article has been deposited with the Crossref Funder Registry. 
2 Clive AO, Bhatnagar R, Psallidas I, et al. Individualised management of malignant pleural effusion. Lancet Respir Med 2015; 3: 505-506.

3 Psallidas I, Kanellakis NI, Gerry S, et al. Development and validation of response markers to predict survival and pleurodesis success in patients with malignant pleural effusion (PROMISE): a multicohort analysis. Lancet Oncol 2018; 19: 930-939.

4 Davies HE, Mishra EK, Kahan BC, et al. Effect of an indwelling pleural catheter versus chest tube and talc pleurodesis for relieving dyspnea in patients with malignant pleural effusion: the TIME2 randomized controlled trial. JAMA 2012; 307: 2383-2389.

5 Psallidas I, Karabela SP, Moschos C, et al. Specific effects of bortezomib against experimental malignant pleural effusion: a preclinical study. Mol Cancer 2010; 9: 56.

6 Rahman NM, Davies HE, Salzberg M, et al. Use of lipoteichoic acid-T for pleurodesis in malignant pleural effusion: a phase I toxicity and dose-escalation study. Lancet Oncol 2008; 9: 946-952.

7 Stathopoulos GT, Zhu Z, Everhart MB, et al. Nuclear factor- $\mathrm{\kappa B}$ affects tumor progression in a mouse model of malignant pleural effusion. Am J Respir Cell Mol Biol 2006; 34: 142-150. 